$\mathrm{UCD}-94-25$

June, 1994

\title{
GAUGE-COUPLING UNIFICATION AND THE MINIMAL SUSY MODEL: A FOURTH GENERATION BELOW THE TOP?
}

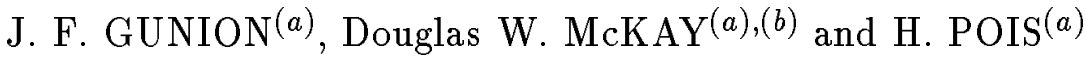 \\ (a) Davis Institute for High Energy Physics, \\ Department of Physics, U. C. Davis, Davis, CA 95616 \\ (b) University of Kansas, Department of Physics and Astronomy, \\ Lawrence, Kansas, 66045
}

\begin{abstract}
We explore the possibility of a fourth generation in the gauge-coupling-unified, minimal supersymmetric (MSSM) framework. We find that a sequential fourth generation (with a heavy neutrino $\nu^{\prime}$ ) can still fit, surviving all present experimental constraints, provided $\lambda_{b}\left(M_{U}\right)=\lambda_{\tau}\left(M_{U}\right)$ Yukawa unification is relaxed. For the theory to remain perturbative up to $M_{U}$, the new leptonic generation must lie within reach of LEP-II and the new $b^{\prime}, t^{\prime}$ must have masses within the reach of the Tevatron. For example, for $m_{t}>150 \mathrm{GeV}$ we find $m_{\nu^{\prime}}, m_{\tau^{\prime}}<86 \mathrm{GeV}, m_{t^{\prime}}<178$, and $m_{b^{\prime}}<156 \mathrm{GeV}$. Experiments at Fermilab are already sensitive to the latter mass regions; we comment on direct $b^{\prime}$ searches and on the $m_{t^{\prime}} \simeq m_{t}$ case in light of new CDF data. Discovery may involve novel decay signatures; however, CDF and LEP-II will confirm or exclude an MSSM fourth generation in the near future.
\end{abstract}

\section{Introduction}

The minimal supersymmetric model (MSSM) prediction of gauge-coupling unification consistent with experiment ${ }^{[1]}$ makes it the most successful approach for relating physics at the unification scale, $M_{U}$, to physics below a $\mathrm{TeV}$, and has led to many detailed investigations of Yukawa and superparticle phenomenology within the MSSM context. ${ }^{[2,3]}$ In this paper we return to an old question that has not been re-examined using the most recent experimental constraints and theoretical inputs. Namely: how much room (if any) is there to add a fourth generation to the "MSSM-plus-unification" framework? ${ }^{\dagger}$ Indeed, aside from occasional studies ${ }^{[4]}$ and experimental searches, broad interest in a possible fourth generation ended abruptly when Mark II and detectors at LEP found that only three light neutrinos are allowed. ${ }^{[5]}$ To provide non-zero mass for the fourth generation $\nu^{\prime}$ in the minimal manner, we introduce an $S U(3) \times S U(2) \times U(1)$ gauge singlet, right-handed neutrino $\nu_{R}{ }^{[6] \ddagger}$ and its corresponding superfield.

\footnotetext{
* Permanent Address.

$\dagger$ We identify the fourth generation by the CKM matrix hierarchy: $V_{t b} \simeq V_{t^{\prime} b^{\prime}}>V_{t^{\prime} b} \sim V_{t b^{\prime}}$.

$\ddagger$ We note that in the minimal $S U(5)$ model, adding a $\nu_{R}$ would lead to a Dirac mass that would naturally be of the order of the quarks or leptons due to the $\mathbf{5}_{\mathbf{f}} \overline{\mathbf{5}}_{\mathbf{H}} \nu_{\mathbf{R}}$ coupling.
} 
Previous works have argued against the inclusion of a fourth generation in the MSSM context on the basis of rather specific assumptions and/or approximations. For instance, if it is assumed that the $\tau$ and $b$ Yukawa couplings unify, i.e. $R_{b / \tau} \equiv \lambda_{b}\left(M_{U}\right) / \lambda_{\tau}\left(M_{U}\right)=1$ (as motivated primarily by the minimal $S U(5)$ GUT theory ${ }^{\S}$ ), inclusion of a fourth generation in the Standard Model (SM) or the MSSM spoils the good (given the uncertainty in $m_{b}{ }^{[7]}$ ) prediction for $m_{b} / m_{\tau}{ }^{[8]}$ obtained in the absence of a fourth generation. Other early studies excluding a fourth generation employed specific parameter choices (often in conjunction with imposing exact Yukawa unification) that limited $m_{t}$ to low values $\left(20 \mathrm{GeV}<m_{t}<120 \mathrm{GeV}\right)$. For example, Bjorkman and Jones ${ }^{[8]}$ assumed $\tan \beta=1$, whereas larger values of $\tan \beta$ must be chosen to allow for a heavier top-quark. We find that if $\tan \beta>1$ is allowed and $R_{b / \tau}=1$ is relaxed (as motivated below), then there is still significant room for a fourth generation "of quarks and leptons (with masses below $m_{t}$ ), and their superpartners.

Relaxation of $\lambda_{b}\left(M_{U}\right)=\lambda_{\tau}\left(M_{U}\right)$ is motivated by recent theoretical work indicating that Yukawa unification at $M_{U}$ is not necessarily expected given possibly large threshold effects at SUSY breaking and $\mathcal{O}\left(M_{U}\right)$ scales, ${ }^{[9]}$ and/or because there may be no symmetry (global and/or local) in the model at $M_{U}$ which leads to an exact equality among the Yukawa couplings; this latter is the case for several candidate supergravity theories (e.g. flipped $S U(5)^{[10]}$ ) and for mild extensions of the minimal $S U(5)$ model.

The shift in focus to large top-quark masses, aside from requiring that $\tan \beta>1$ be considered, has led many authors to promote the naturalness of infrared 'fixedpoint' solutions to the RG equations, ${ }^{[12]}$ for which a large range of top Yukawacoupling $\left(\lambda_{t}\right)$ values evolves down to a narrow range of large values at the weak scale; a close correlation between $\tan \beta$ and $m_{t}$ arises. We will discuss whether or not such approximate 'fixed-point' behavior for some or all of the quark Yukawas of the extra generation can be realized.

Our study has immediate experimental relevance. The requirement that all couplings remain perturbative places restrictive upper bounds on the $t^{\prime}, b^{\prime}, \nu^{\prime}$ and $\tau^{\prime}$ masses. These are such that the question of an MSSM fourth generation may be settled in the next few years by searches at the Tevatron for an appropriate set of new signatures (i.e. besides $t \rightarrow b+W$ ). And certainly LEP-II will allow detection of direct $\tau^{\prime}+\bar{\tau}^{\prime}$ and/or $\nu^{\prime}+\bar{\nu}^{\prime}$ production for masses up to the maximum allowed.

$\S$ By 'minimal' we mean the standard $S U(5) \mathbf{5}, \overline{\mathbf{5}}, 24$ Higgs representation content.

I More than four generations are clearly excluded. $N_{g}>4$ in the MSSM results in loss of asymptotic freedom for $S U(3)$ color, and inability to evolve perturbatively to a point of gauge-coupling unification; see Bjorkman and Jones, Ref. [8].

* For example, $R_{b / \tau} \neq 1$ in general when a $\mathbf{4 5}$ Higgs representation ${ }^{[11]}$ is added. 


\section{The Renormalization Group Equations and Procedure ${ }^{* *}$}

For a sequential fourth generation model with the addition of a gauge singlet $\nu_{R}$ the one-loop predictions for $M_{U}$ and $\alpha_{3}\left(m_{Z}\right)$ are independent of the number of generations, and there is only a mild dependence of $M_{U}$ and $\alpha_{3}\left(m_{Z}\right)$ on $N_{g}$ at two-loops, implying that the gauge-coupling sector alone will not restrict $N_{g}$.

In contrast, $N_{g}$ does affect Yukawa coupling unification at one loop. In order to accommodate a massive $\nu^{\prime}$ we introduce the superpotential Yukawa interaction $\delta \mathcal{W} \equiv \lambda_{\nu^{\prime}} \hat{L}^{\prime} \hat{H}_{2} \hat{\nu}_{R}^{\prime}$ where $\hat{L}^{\prime}=\left(\hat{\nu}^{\prime}, \hat{\tau}^{\prime}\right)_{L}$ and $\hat{H}_{2}$ is the Higgs doublet superfield whose neutral scalar component gives mass to up-type quarks and (now) the $\nu^{\prime}$. The RG equations obtained in the absence of the $\nu^{\prime[13]}$ are modified by: i) the RGE for the fourth generation neutrino $y_{\nu^{\prime}}$; and ii) the additional $y_{\nu^{\prime}}$ terms which appear in the RGE's for $y_{t}$ and $y_{t^{\prime}}$ (via Higgs field renormalization) and for $y_{\tau^{\prime}}$ (via fermion field renormalization) - here, we have defined $y_{f} \equiv \lambda_{f}^{2} / 4 \pi$. More explicitly, for the third and fourth generation fermions we obtain (at one-loop):

$$
\frac{d y_{i}}{d t}=\left(A_{j i} y_{j}-\alpha_{k} B_{k i}\right) y_{i}, \quad i=\text { fixed }
$$

where $i, j=\left(t, b, \tau, t^{\prime}, b^{\prime}, \nu^{\prime}, \tau^{\prime}\right)=(1,2,3,4,5,6,7),\left(\alpha_{k}\right)=\left(\alpha_{1}, \alpha_{2}, \alpha_{3}\right)$ (where $\alpha_{i} \equiv$ $\left.g_{i}^{2} / 4 \pi\right), t \equiv \ln (\mu) / 2 \pi$ and the $A_{j i}, B_{k i}$ matrices are

$$
A=\left(\begin{array}{ccccccc}
6 & 1 & 0 & 3 & 0 & 3 & 0 \\
1 & 6 & 3 & 0 & 3 & 0 & 3 \\
0 & 1 & 4 & 0 & 1 & 0 & 1 \\
3 & 0 & 0 & 6 & 1 & 3 & 0 \\
0 & 3 & 3 & 1 & 6 & 0 & 3 \\
1 & 0 & 0 & 1 & 0 & 4 & 1 \\
0 & 1 & 1 & 0 & 1 & 1 & 4
\end{array}\right), \quad B=\left(\begin{array}{ccccccc}
\frac{13}{15} & \frac{7}{15} & \frac{9}{5} & \frac{13}{15} & \frac{7}{15} & \frac{3}{5} & \frac{9}{5} \\
3 & 3 & 3 & 3 & 3 & 3 & 3 \\
\frac{16}{3} & \frac{16}{3} & 0 & \frac{16}{3} & \frac{16}{3} & 0 & 0
\end{array}\right)
$$

Since $\lambda_{u, d}=\sqrt{2} m_{u, d} /[v(\sin \beta, \cos \beta)]$ (with $\left.v=246 \mathrm{GeV}, \tan \beta \equiv v_{2} / v_{1}\right)$, and fourth generation masses must satisfy the LEP bounds $m_{u, d}>m_{Z} / 2$, all of the fourth generation Yukawa couplings must be relatively large at the weak scale: $\lambda_{i} \gtrsim 0.25$. As a result, the Yukawas only remain perturbative during evolution up to $M_{U}$ for a severely limited range of $\tan \beta, m_{t}, m_{t^{\prime}}, m_{b^{\prime}}, m_{\nu^{\prime}}$ and $m_{\tau^{\prime}}$. Indeed, we find that $\tan \beta$ must be $\lesssim 3$ in order to avoid singular Yukawa coupling behavior;

** We assume that the third and fourth generations are unmixed, but later comment briefly on the (small) effects of allowing arbitrary mixing. 
large $\tan \beta$ solutions are not possible when a fourth generation is included. When the values of $m_{t}, m_{t^{\prime}}, m_{b^{\prime}}, m_{\nu^{\prime}}, m_{\tau^{\prime}}$ are comparable, the $y_{\tau^{\prime}}$ and $y_{\nu^{\prime}}$ couplings in Eq. 1 are the most susceptible to singular "Landau-pole" behavior since there is no large, negative gluon $\left(\alpha_{3}\right)$ term in their $\beta$-functions to control the growth driven by the large, positive Yukawa terms. Only when $m_{t}$ is large and $m_{\nu^{\prime}, \tau^{\prime}}$ are near the LEP lower bound of $\sim m_{Z} / 2$ (see Sec. 4) do other Yukawas first go nonperturbative. Then, the (large) $t, t^{\prime}, b^{\prime}$ Yukawas feed one another's growth, and all receive additional boosts from the $\nu^{\prime}$ and $\tau^{\prime}$ Yukawas, with the result that $\lambda_{t}, \lambda_{b^{\prime}}$ or $\lambda_{t^{\prime}}$ can go non-perturbative sooner than $\lambda_{\tau^{\prime}, \nu^{\prime}}$ in evolving up to $M_{U}$.

Although the fourth generation Yukawa factor $3 y_{b^{\prime}}+y_{\tau^{\prime}}$ appears in the $\beta$ functions for $y_{b}$ and $y_{\tau}$ in Eq. 1, this common fourth-generation down-sector (Higgs field renormalization) term cancels in $\frac{d}{d t}\left(\frac{y_{b}}{y_{\tau}}\right)$ yielding a result identical to the three generation case. Nonetheless, the presence of a fourth generation tends to drive $m_{b}$ to unacceptably high values if $R_{b / \tau}=1$ is required. ${ }^{[8]}$ To see why, first recall that quark to lepton Yukawa ratios that are of $\mathcal{O}(1)$ at $M_{U}$ evolve to values that are larger than one as one runs down to the weak scale (and below) due to the negative $\alpha_{3}$ terms that are present in the quark Yukawa RGE's but absent from lepton Yukawa RGE's. This effect is substantially magnified for $N_{g}=4$ compared to $N_{g}=3$ because gauge unification yields larger $\alpha_{U}$, and hence $\alpha_{3}\left(t<M_{U}\right)$, when $N_{g}=4$. The indirect influence of $y_{t^{\prime}}$ and $y_{\nu^{\prime}}$ on $y_{t}$ also affects the low-energy $m_{b} / m_{\tau}$ value predicted for a given choice of $\lambda_{b} / \lambda_{\tau}$ at $M_{U}$, but cannot compensate for the $\alpha_{3}$ magnification effect. Evolution of $R_{b^{\prime} / \tau^{\prime}}\left(\equiv \lambda_{b^{\prime}}\left(M_{U}\right) / \lambda_{\tau^{\prime}}\left(M_{U}\right)\right)$ involves the fourth generation couplings directly, and it is possible to have $R_{b^{\prime}} / \tau^{\prime}=1$ for sufficiently small $\tan \beta$. In any case, as motivated in the introduction, we shall not require $b / \tau$ and/or $b^{\prime} / \tau^{\prime}$ unification.

\section{Two-Loop RG Equation Results}

At two-loop order, the RG equations in both the gauge and Yukawa sectors are fully coupled. For this study, two-loop Yukawa RG equations are used, but in the gauge sector only the two-loop mutual gauge couplings are retained. (The two-loop Yukawa effects on the gauge sector are small and will be commented upon shortly.) Further, we assume that the SUSY mass scale and $m_{Z}$ are the same, so that there is no intermediate decoupling of SUSY partner fields. Gauge-coupling unification along with the experimental central values of $\sin ^{2} \theta_{W}\left(m_{Z}\right)=0.2324^{[14]}$ and $\alpha_{Q E D}\left(m_{Z}\right)=1 / 127.9$ then determines $\alpha_{3}\left(m_{Z}\right)$; the result is $\alpha_{3}\left(m_{Z}\right)=0.130$. We also obtain $M_{U}=5.46 \times 10^{16} \mathrm{GeV}$ and $\alpha_{U}=0.0936{ }^{[14]}$ The Yukawa equations are then solved iteratively subject to requiring definite input values of $\tan \beta$ and $m_{t}\left(m_{t}\right), m_{b}\left(m_{b}\right), m_{\tau^{\prime}}\left(m_{\tau^{\prime}}\right)$, and $m_{\nu^{\prime}}\left(m_{\nu^{\prime}}\right)$. A bound of $\lambda_{f}<3$ is imposed in order to guarantee perturbative validity for the RGE's as defined by a modest ratio of two-loop to one-loop effects. ${ }^{[15]}$ The output comprises values of $m_{b^{\prime}}\left(m_{b^{\prime}}\right)$ and $m_{t^{\prime}}\left(m_{t^{\prime}}\right)$ that are consistent with the RGE's and the perturbative bounds. An

* Unless otherwise noted, all masses referred to in the following are the running mass $m(m)$. 


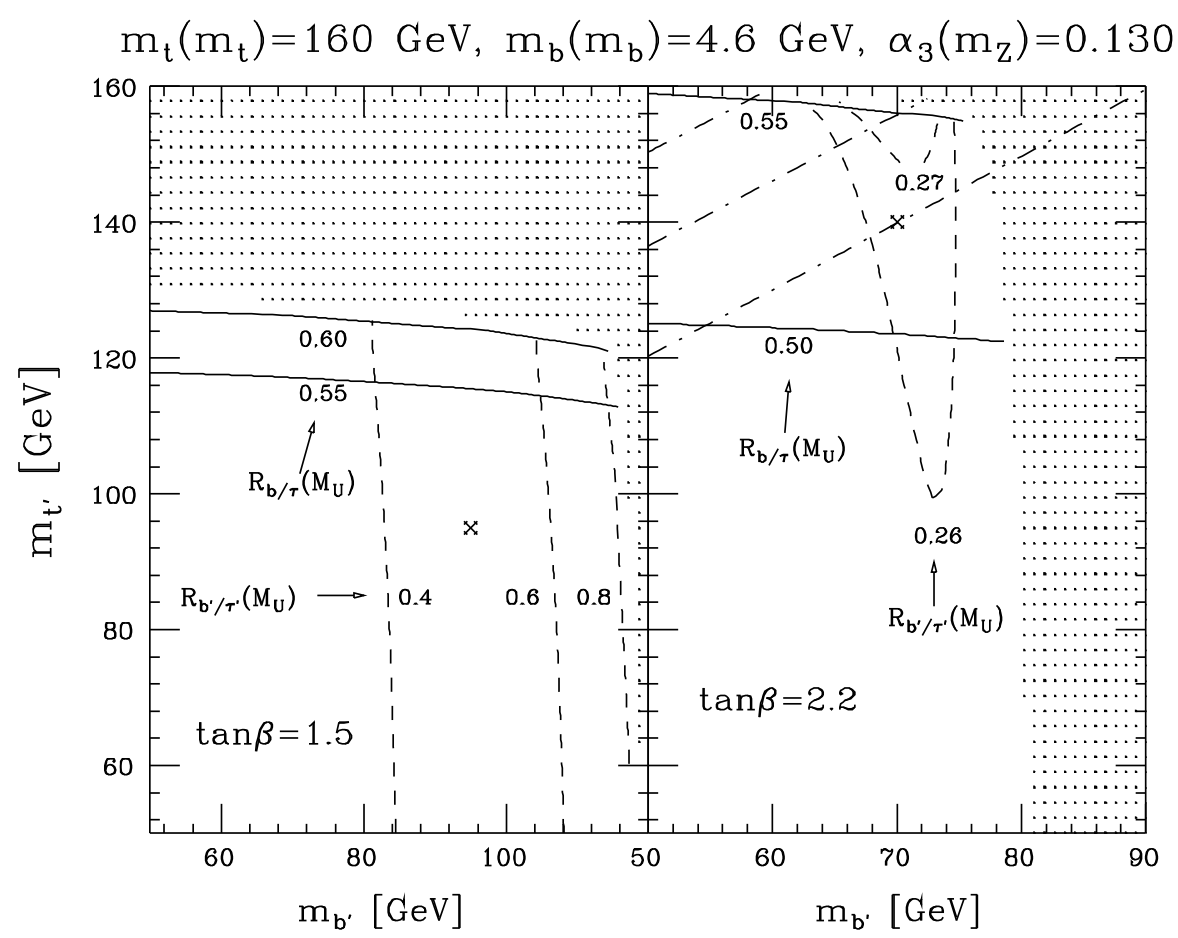

Figure 1: The allowed region in $m_{b^{\prime}}-m_{t^{\prime}}$ parameter space is illustrated for $m_{\tau^{\prime}}=$ $m_{\nu^{\prime}}=50 \mathrm{GeV}, m_{b}=4.6 \mathrm{GeV}$, and $m_{t}=160 \mathrm{GeV}$. (All masses are $m(m)$ running masses.) The perturbatively disallowed region is dotted. Within the allowed undotted region, the horizontal solid (vertical dashed) lines correspond to fixed values of $R_{b / \tau}\left(R_{b^{\prime} / \tau^{\prime}}\right)$ as labelled. Results for $\tan \beta=1.5$ and 2.2 are shown. The dash-dot lines in the $\tan \beta=2.2$ window are contours of $\Delta \rho=0.002,0.004$ and 0.006 (in order of decreasing restrictiveness). The two-loop Yukawa corrections discussed for Table 1 were evaluated at the points marked by the $x$ 's.

allowed choice for the $t^{\prime}$ and $b^{\prime}$ running masses yields definite values for $R_{b / \tau}$ and $R_{b^{\prime} / \tau^{\prime}}$

As discussed in Sec. 2, $\lambda_{\nu^{\prime}}$ and $\lambda_{\tau^{\prime}}$ are intrinsically the most susceptible to Landau-pole divergence in evolving up to $M_{U}$. Indeed, $\lambda_{\nu^{\prime}}$ or $\lambda_{\tau^{\prime}}$ are typically driven to non-perturbative values unless $m_{\nu^{\prime}}, m_{\tau^{\prime}}$ are near their lower bound of $\sim m_{Z} / 2$. Thus, to explore large $t, t^{\prime}, b^{\prime}$ Yukawas and large $m_{b^{\prime}, t^{\prime}}$ values, we employ large $m_{t}$, take $m_{\tau^{\prime}}=m_{\nu^{\prime}}=50 \mathrm{GeV}$, and examine the region of perturbatively allowed solutions in the $m_{b^{\prime}}-m_{t^{\prime}}$ plane for various values of $\tan \beta$.

Fig. 1 illustrates the scope of the perturbatively-allowed solutions, adopting $m_{b}=4.6 \mathrm{GeV}^{\dagger}$ and $m_{t}=160 \mathrm{GeV}$ (i.e. $m_{t}($ pole $\left.)=169 \mathrm{GeV}\right) .+\frac{\ddagger}{\dagger}$ For $\tan \beta=1.5$ we find a nearly square allowed region extending to $m_{b^{\prime}}, m_{t^{\prime}} \lesssim 120-125 \mathrm{GeV}$, as

$\dagger$ Upper limits of $m_{b}\left(m_{b}\right)$ allowed in Refs. $[9,10]$ range from 4.45 to $4.9 \mathrm{GeV}$.

$\ddagger$ Similar results apply for higher $m_{t}$. 
shown in Fig. 1a. For $\tan \beta=2.2$ (and higher ${ }^{\S}$ ), larger $m_{t^{\prime}}$ values $\lesssim m_{t}$ are possible but only for $m_{b^{\prime}}<m_{Z}$, as illustrated in Fig. 1b. For both $\tan \beta$ cases, $R_{b / \tau}, R_{b^{\prime} / \tau^{\prime}}$ are simultaneously near-maximal (but $<1$ ) in the large- $m_{b^{\prime}}$, large- $m_{t^{\prime}}$ corner of parameter space. In Fig. 1a, $m_{t^{\prime}}^{\max }$ at fixed $m_{b^{\prime}}\left(m_{b^{\prime}}^{\max }\right.$ at fixed $\left.m_{t^{\prime}}\right)$ occurs when $\lambda_{t}$ $\left(\lambda_{b^{\prime}}\right.$, with $\lambda_{\tau^{\prime}}$ close behind) goes non-perturbative. In Fig. 1b, $m_{t^{\prime}}^{\max }$ occurs when $\lambda_{t^{\prime}}$ (followed very closely by $\lambda_{\nu^{\prime}}$ and $\lambda_{t}$ ) goes non-perturbative; at $m_{b^{\prime}}^{\max }$ it is $\lambda_{\tau^{\prime}}$ that goes non-perturbative. For higher $m_{\nu^{\prime}}=m_{\tau^{\prime}}($ e.g. $\gtrsim 55 \mathrm{GeV}$ at $\tan \beta=1.5)$ it is $\lambda_{\tau^{\prime}}$ that goes non-perturbative at all boundary points.

The heavy neutrino effects, included here for the first time in this type of study, typically lower the $m_{t^{\prime}}$ upper limit by about $5 \mathrm{GeV}$ for given input values for $m_{t}$, $\tan \beta$ and $m_{\tau^{\prime}}$. For specific allowed values of $m_{t^{\prime}}$ and $m_{b^{\prime}}$ they cause the value of $R_{b / \tau}\left(R_{b^{\prime}} / \tau^{\prime}\right)$ to rise by $3-5 \%(20-30 \%)$ compared to the massless neutrino case. Thus, the $\nu^{\prime}$ couplings do not change the qualitative picture, but they are certainly not negligible.

Table 1: Corrections to $\alpha_{3}\left(m_{Z}\right), \alpha_{U}, M_{U}, R_{b / \tau}$ and $R_{b^{\prime} / \tau^{\prime}}$ due to two-loop Yukawa effects in the gauge sector for the selected points shown by the $x^{\prime}$ 's in Fig. 1 (with $m_{\nu^{\prime}, \tau^{\prime}}=50 \mathrm{GeV}$ and $m_{t}=160 \mathrm{GeV}$ ).

The 0 superscript indicates values before including two-loop effects.

\begin{tabular}{c|c|c|c|c|c}
\hline \hline$\left(m_{t^{\prime}}, m_{b^{\prime}}\right)(\mathrm{GeV})$ & $\alpha_{3} / \alpha_{3}^{0}$ & $\alpha_{U} / \alpha_{U}^{0}$ & $M_{U} / M_{U}^{0}$ & $R_{b / \tau} / R_{b / \tau}^{0}$ & $R_{b^{\prime} / \tau^{\prime}} / R_{b^{\prime} / \tau^{\prime}}^{0}$ \\
\hline$(95,95)(\mathrm{GeV})$ & 0.97 & 0.95 & 0.91 & 1.08 & 1.05 \\
\hline$(140,70)(\mathrm{GeV})$ & 0.96 & 0.94 & 0.91 & 1.11 & 1.06 \\
\hline \hline
\end{tabular}

Table 1 indicates the effects of including two-loop Yukawa terms in the gaugecoupling RGE's at the points marked by $X^{\prime}$ 's in Fig. 1 . Since $\alpha_{3}$ is decreased, allowed solution regions are slightly reduced and $R_{b / \tau}$ and $R_{b^{\prime} / \tau^{\prime}}$ are increased by up to $10 \%$.

Figure 2a gives the maximum allowed $m_{t^{\prime}}\left(m_{b^{\prime}}\right)$ values as a function of $\tan \beta$, for $m_{b}\left(m_{b}\right)=4.6 \mathrm{GeV}$ and $m_{t}\left(m_{t}\right)=160 \mathrm{GeV}$; these are attained by setting $m_{b^{\prime}}=45 \mathrm{GeV}\left(m_{t^{\prime}}=45 \mathrm{GeV}\right)$, respectively. Results are shown for the two choices $m_{\tau^{\prime}}=m_{\nu^{\prime}}=50$ and $75 \mathrm{GeV}$. Note that $m_{b^{\prime}}^{\max }$ is attained just above the lower $\tan \beta$ limit where $m_{t^{\prime}}^{\max }$ is forced below $45 \mathrm{GeV}$, while $m_{t^{\prime}}^{\max }$ occurs near the upper $\tan \beta$ end-point where $m_{b^{\prime}}^{\max }$ is forced below $45 \mathrm{GeV}$. For $m_{\tau^{\prime}}, m_{\nu^{\prime}}=50 \mathrm{GeV}$ (as in Fig. 1), Fig. 2 a shows that non-perturbative values for all the $\lambda_{i}$ can only be avoided for $1 \lesssim \tan \beta \lesssim 3$. The LEP bounds on the fourth generation are crucial in arriving at this very restrictive limit of $\tan \beta \lesssim 3$. (The 3 -generation-like $\tan \beta$ range, $\tan \beta \lesssim 60$, is only attained in the phenomenologically disallowed limit where all

$\S$ We will shortly establish an upper limit of $\tan \beta \lesssim 3$.

I We have solved the one-loop RG equations for the case of arbitrary 3-4 generation mixing, and find effects on the boundaries and the values of $R_{b / \tau}$ at only the few percent level. 


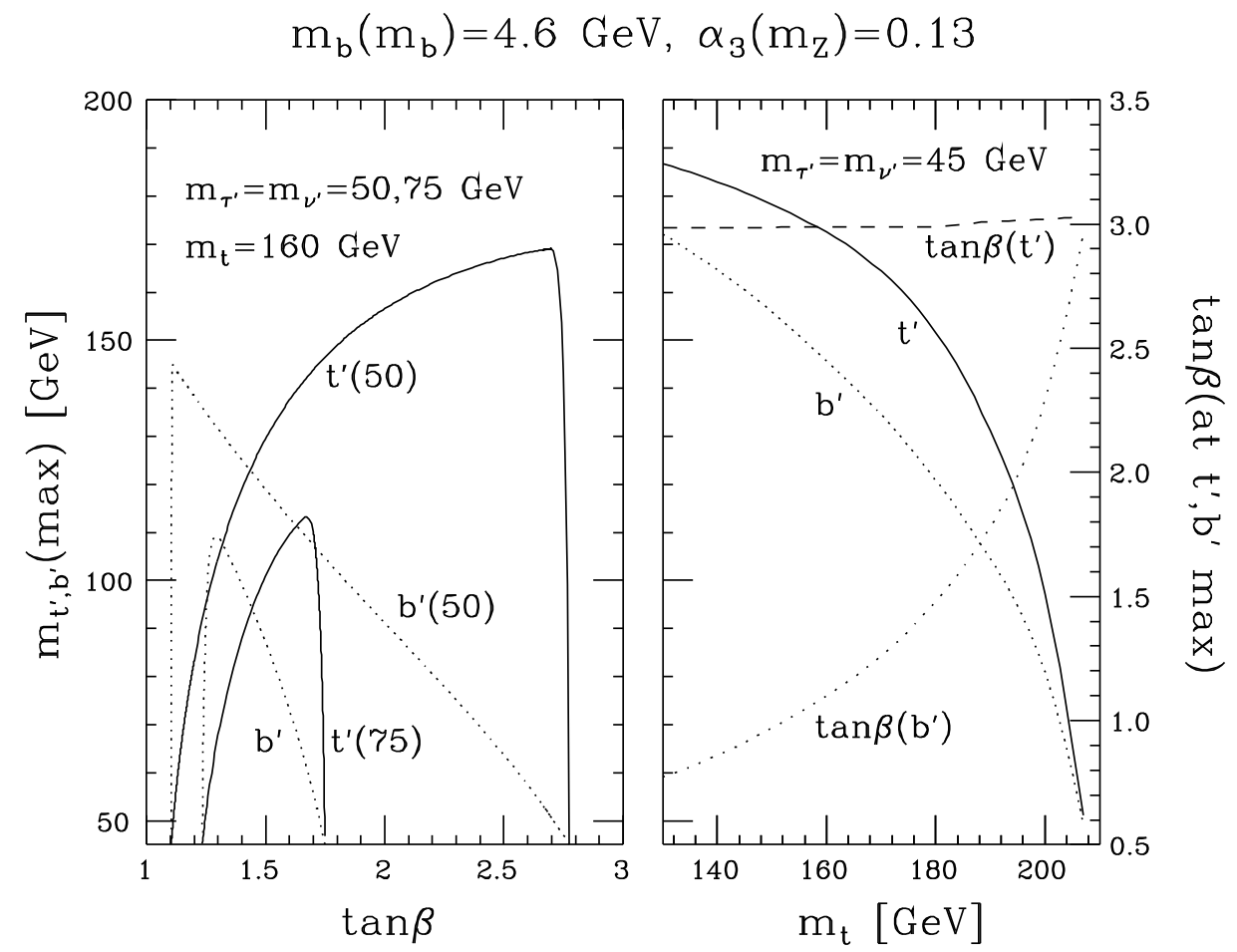

Figure 2: In a), we display the maximal allowed values for $m_{t^{\prime}}, m_{b^{\prime}}$ as a function of $\tan \beta$ taking $m_{b}\left(m_{b}\right)=4.6 \mathrm{GeV}, m_{t}\left(m_{t}\right)=160 \mathrm{GeV}$ (corresponding to $m_{t}$ (pole) $=$ $169 \mathrm{GeV}$ ), and $m_{\tau^{\prime}}=m_{\nu^{\prime}}=50$ or $75 \mathrm{GeV}$ (as indicated by the numbers in parenthesis): the solid (dotted) curves correspond to $m_{t^{\prime}}^{\text {max }}$ ( $m_{b^{\prime}}^{\text {max }}$ ) assuming $m_{b^{\prime}}=45 \mathrm{GeV}\left(m_{t^{\prime}}=45 \mathrm{GeV}\right)$. In b), we take $m_{\tau^{\prime}}=m_{\nu^{\prime}}=45 \mathrm{GeV}$. The solid (densely dotted) curves give $m_{t^{\prime}}^{\max }\left(m_{b^{\prime}}^{\max }\right)$ as a function of $m_{t}\left(m_{t}\right)$; the dashed (sparsely dotted) curves show (on the right-hand $y$-axis) the value of $\tan \beta$ at which these respective maximal values are attained.

fourth-generation masses - and, hence, Yukawas - can be simultaneously near zero, leaving only the $N_{g}=4$ effect in the gauge RG equations.) As $m_{\tau^{\prime}}, m_{\nu^{\prime}}$ increase, the allowed $\tan \beta$ domain collapses further and $m_{t^{\prime}}^{\text {max }}$ and $m_{b^{\prime}}^{\max }$ quickly fall towards $m_{Z} / 2$, as illustrated by the $m_{\tau^{\prime}}=m_{\nu^{\prime}}=75 \mathrm{GeV}$ curves in Fig. $2 \mathrm{a}$. At $m_{t}=160 \mathrm{GeV}, m_{\tau^{\prime}}=m_{\nu^{\prime}}>83 \mathrm{GeV}$ is excluded if we require $m_{t^{\prime}}, m_{b^{\prime}} \geq 45 \mathrm{GeV}$.

Of course, the allowed solution regions vary with $m_{t}$. The absolute maximal values of $m_{t^{\prime}}$ and $m_{b^{\prime}}$ consistent with LEP limits (found by setting $m_{\tau^{\prime}}, m_{\nu^{\prime}}=$ $45 \mathrm{GeV}$ and scanning in $\tan \beta$ as in Fig. $2 \mathrm{a}$ ), and the $\tan \beta$ values at which they are attained, are plotted in Fig. $2 \mathrm{~b}$ as a function of $m_{t}$. We see that the highest value of $m_{t}\left(m_{t}\right)$ for which there is a consistent perturbative solution to the gaugecoupling and Yukawa RGE's is $m_{t} \sim 207 \mathrm{GeV}$. At this $m_{t}, m_{b^{\prime}}^{\max }$ and $m_{t^{\prime}}^{\max }$ are simultaneously forced to the imposed $45 \mathrm{GeV}$ LEP limit and the $\tan \beta$ values at which these maxima are achieved converge to a value just above 3 . As $m_{t}$ is decreased, $m_{b^{\prime}}^{\max }$ and $m_{t^{\prime}}^{\max }$ both rise rapidly and the $\tan \beta$ value at which $m_{b^{\prime}}^{\max }$ is attained falls rapidly relative to the rather constant $\tan \beta \sim 3$ value at which 
$m_{t^{\prime}}^{\max }$ is attained. Note that $m_{t^{\prime}}>m_{t}$ becomes possible for $m_{t} \lesssim 170 \mathrm{GeV}$. In analogous fashion, we determine the maximum allowed $m_{\tau^{\prime}}=m_{\nu^{\prime}}$ value subject to requiring $m_{t^{\prime}}, m_{b^{\prime}}>45 \mathrm{GeV}$. The results for $m_{t}=130,150,170,190,207 \mathrm{GeV}$ are $m_{\tau^{\prime}}=m_{\nu^{\prime}}=90.7,86.1,79.0,67.9,45.0 \mathrm{GeV}$, respectively. These maxima are achieved at $\tan \beta=1.26,1.37,1.56,1.94$ and 3.02 , respectively.

Finally, let us discuss whether or not some or all of the Yukawa couplings are naturally near their fixed-point (defined by zero derivative) at scale $m_{Z}$. In the case of three generations, requiring $R_{b / \tau}=1$ (and $m_{b}\left(m_{b}\right)=4.25 \mathrm{GeV}$ ) implied that $m_{t}$ was close to its $\left(\tan \beta\right.$-dependent) fixed-point value at $m_{Z}$, and evolved to large values at $M_{U}{ }^{[12,15]}$ This connection between fixed-point behavior and Yukawa unification is modified for four generations. We have already noted that $R_{b / \tau}<1$ for all perturbatively allowed $m_{t}, m_{t^{\prime}}, m_{b^{\prime}}$ choices. Nonetheless, for small enough $\tan \beta, m_{\nu^{\prime}}$, and $m_{\tau^{\prime}}$ choices, $m_{t}, m_{t^{\prime}}, m_{b^{\prime}}$ values within the perturbative domain can be found such that $\lambda_{t}, \lambda_{t^{\prime}}, \lambda_{b^{\prime}}$ are all simultaneously very close to their fixed-point values. For $m_{\nu^{\prime}}=m_{\tau^{\prime}}=50 \mathrm{GeV}$ and $\tan \beta=1.5$ the $\lambda_{t}, \lambda_{t^{\prime}}$ and $\lambda_{b^{\prime}}$ derivatives

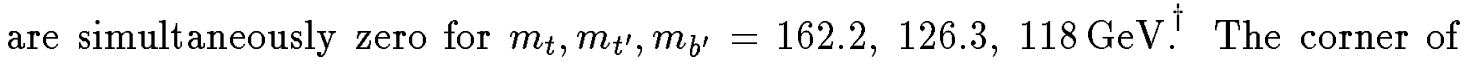
the $\tan \beta=1.5, m_{t}=160 \mathrm{GeV}$ Fig. $1 \mathrm{a}$ where $m_{b^{\prime}}, m_{t^{\prime}}, R_{b / \tau}$ and $R_{b^{\prime} / \tau^{\prime}}$ are all near maximal is very close to the $\tan \beta=1.5$ fixed-point solution. For still smaller $\tan \beta$, one can achieve $R_{b^{\prime} / \tau^{\prime}} \sim 1$ and somewhat larger $R_{b / \tau}$. For example, for $\tan \beta=1.2$ and $m_{t}=160 \mathrm{GeV}$ the parameter-space-corner point $m_{t^{\prime}}, m_{b^{\prime}}=78,136 \mathrm{GeV}$ yields $R_{b / \tau}, R_{b^{\prime} / \tau^{\prime}}=0.65,1.1$. Such points are even closer to a $\lambda_{t}, \lambda_{t^{\prime}}, \lambda_{b^{\prime}}$ fixed point. In contrast, for $\tan \beta \gtrsim 2$ it is not possible to achieve large $R_{b^{\prime}} / \tau^{\prime}$ or approach fixedpoint behavior; a small $R_{b^{\prime} / \tau^{\prime}}$ value implies a $\lambda_{b^{\prime}}$ value well below 1 as $\lambda_{\tau^{\prime}}$ passes (as $m_{b^{\prime}}$ is increased) the perturbative limit of 3 . In general, adjusting $m_{t}, m_{t^{\prime}}, m_{b^{\prime}}$ so that both $R_{b / \tau}$ and $R_{b^{\prime} / \tau^{\prime}}$ are as large as perturbatively allowed at a given $\tan \beta$ implies that $\lambda_{t}, \lambda_{t^{\prime}}, \lambda_{b^{\prime}}$ will all be as close to $m_{Z^{-}}$-scale fixed-point values as perturbatively possible - however, the fixed point lies increasingly beyond the perturbative domain as $\tan \beta$ is increased (which forces $\lambda_{\tau^{\prime}}$ to reach its perturbative limit of 3 at $m_{b^{\prime}}^{\max }$ while other Yukawas are still small).

\section{Experiments and a Fourth Generation}

The bound $m_{f} \gtrsim m_{Z} / 2$ on any new sequential fermion, quark or lepton, that has been set at $\mathrm{LEP}^{[16]}$ is the firmest experimental constraint on the fourth generation masses. A full analysis of constraints on the masses of fourth family quarks with small mixings to lower-mass families from precision electroweak observables

* $\lambda_{\tau^{\prime}}$ and $\lambda_{\nu^{\prime}}$ cannot be at a fixed point at scale $m_{Z}$ since all fourth-generation Yukawas must be large (due to LEP and other lower bounds on the associated masses) and since there is no large $\alpha_{3}$ term to cancel the large Yukawa coupling terms in the $y_{\tau^{\prime}}$ and $y_{\nu^{\prime}}$ evolution equations.

$\dagger$ In general, specifying $\tan \beta, m_{\tau^{\prime}}, m_{\nu^{\prime}}$ yields three equations in the three unknown $\lambda_{t}, \lambda_{t^{\prime}}$ and $\lambda_{b^{\prime}}$ Yukawas; solving yields definite values for the low-scale $t, t^{\prime}, b^{\prime}$ Yukawas and, hence, masses. 
might prove useful. ${ }^{[17 \hbar}$ Indeed, requiring $\Delta \rho\left(t^{\prime}-b^{\prime}\right) \lesssim 0.002^{\S}$ (i.e. $\Delta T \lesssim 0.25$ ), would eliminate the large- $m_{t^{\prime}}$ small- $m_{b^{\prime}}$ corner of the $m_{t}=160 \mathrm{GeV}$ parameter space - see the contour in Fig. 1b. Also, a roughly degenerate fourth family with masses $\gtrsim m_{Z}$ yields $\Delta S \sim 2 / 3 \pi$, a shift comparable to that obtained by changing the Higgs mass from $100 \mathrm{GeV}$ to $1 \mathrm{TeV}$ in the SM. However, because the MSSM RGE perturbative limits imply (correlated) upper bounds on the $b^{\prime}$ and $t^{\prime}$ masses, direct experimental constraints from the Tevatron can be much more significant, depending upon the relative size of the $t^{\prime}$ and $b^{\prime}$ masses.

We consider first the case of $m_{t^{\prime}}>m_{b^{\prime}}$. Constraints on the $b^{\prime}$ depend greatly upon the manner in which it decays. If the $b^{\prime}$ has significant mixing with the 2 nd or 1 st generation then $b^{\prime} \rightarrow c, u+W$ (where the $W$ may be real or virtual) decays will be dominant. The published CDF collaboration data ${ }^{[18]}$ limits the mass of a $b^{\prime}$ that decays primarily to a $c(u)$ quark: $m_{b^{\prime}}>85 \mathrm{GeV}$ at $95 \%$ C.L., using the same $e \mu+X$ final state search that excluded the $t$ in this mass range. Their latest data ${ }^{[19]}$ in the dilepton channel probably can be used to increase this limit. However, if unmixed with lower generations, the $b^{\prime}$ will decay via flavorchanging neutral current (FCNC) channels: $b^{\prime} \rightarrow b \gamma, b g$ or $b Z^{*}$ for $m_{b^{\prime}}<m_{Z}$, with $b^{\prime} \rightarrow b Z$ becoming dominant for $m_{b^{\prime}}>m_{Z}{ }^{*}$. These decays yield distinctly different signatures than the lepton-plus-jet signatures of charged current decays that are already mostly excluded. New search strategies are required. ${ }^{[21]}$ For example, when $m_{b^{\prime}}<m_{Z}$ the process $p \bar{p} \rightarrow b^{\prime} \bar{b}^{\prime} \rightarrow b \gamma+\bar{b} \gamma$ yields a significant rate for 2 jet $+2 \gamma$ final states. Also present, and at a higher rate such that one might be able to afford to $b$-tag, would be $\gamma+3$ jet final states. Present CDF data in combination with the data from the ongoing run may combine to set rather firm $b^{\prime}$ limits. ${ }^{[22]}$ If $m_{b^{\prime}}>m_{Z}$, but below our upper limits of roughly $140-150 \mathrm{GeV}$, then $b^{\prime} \bar{b}^{\prime} \rightarrow b \bar{b} Z Z$ yields a significant excess in the rate for real $Z$ production. For example, if $m_{b^{\prime}}=130 \mathrm{GeV}$ and $B R\left(b^{\prime} \rightarrow b Z\right) \sim 0.8$, there are roughly $60 b^{\prime} b^{\prime}$ events for $L=19.3 \mathrm{pb}^{-1}$ in which at least one of the $Z$ 's decays to $e^{+} e^{-}$or $\mu^{+} \mu^{-}$. Interestingly, in Ref. [19] it is stated that there is a slight excess of $Z$ 's (in association with a tagged $b$ ) that is not inconsistent with production and FCNC decay of a $b^{\prime}$ with $m_{b^{\prime}} \sim 150 \mathrm{GeV}$.

Consider next the $t^{\prime}$. If $m_{t^{\prime}}$ is significantly larger than $m_{b^{\prime}}$, e.g. $m_{t^{\prime}} \simeq m_{t} \gtrsim$ $150 \mathrm{GeV}$ and $m_{b^{\prime}} \lesssim 75 \mathrm{GeV}$ (as in the corner region of Fig. $1 \mathrm{~b}$ ), then $m_{t^{\prime}}$ lies above the published $D 0$ limit on $m_{t}{ }^{[23]}$ and is in the region favored for $m_{t}$ by the recent CDF analysis. ${ }^{[19]}$ The corresponding $t^{\prime}$ production and decay process is especially interesting, giving a complex set of possible final states with six and eight jets. We have performed a rough Monte Carlo study of the number of $t^{\prime} t^{\prime}$ production

$\ddagger$ Contributions from spin-0 superpartners are typically much smaller.

$\S$ The $\tau^{\prime}$ and $\nu^{\prime}$ are close to the lower bound $m_{Z} / 2$ and are ignorable for our purposes.

II In the following experimental constraint discussion, the masses referred to should be thought of as pole masses.

* We temporarily ignore the decays $b^{\prime} \rightarrow h b$ (where $h$ is the light MSSM Higgs boson) that would dominate if allowed. ${ }^{[20]}$ 
Table 2: For $L=19.3 \mathrm{pb}^{-1}$, we tabulate the number of $t^{\prime} t^{\prime}$

events passing our approximations to the CDF top-quark

discovery cuts in the single lepton $+b$-tag and dilepton modes.

\begin{tabular}{c|cccc|cccc}
\hline \hline & \multicolumn{3}{|c|}{ Lepton $+b$-tag Mode } & \multicolumn{5}{c}{ Dilepton Mode } \\
\hline & \multicolumn{4}{|c|}{$m_{b^{\prime}}(\mathrm{GeV})$} & \multicolumn{5}{c}{$m_{b^{\prime}}(\mathrm{GeV})$} \\
$m_{t^{\prime}}(\mathrm{GeV})$ & 50 & 80 & 110 & 130 & 50 & 80 & 110 & 130 \\
\hline 160 & 2.8 & 2.2 & 0.5 & 0.2 & 0.7 & 0.5 & 0.06 & 0.004 \\
130 & 7.3 & 2.8 & 0.07 & - & 1.2 & 0.3 & 0.0006 & - \\
100 & 7.9 & 0.5 & - & - & 0.8 & 0.003 & - & - \\
\hline \hline
\end{tabular}

and decay events in the dilepton-plus-jets and lepton-plus-jets channels (keeping only those final states that do not contain any extra leptons or photons from the $b^{\prime}$ decays), using the quoted CDF luminosity and approximating their cuts and $b$-tagging procedures. Branching ratios of $b^{\prime}$ decays are estimated from Ref. [21]. A summary of the predicted event rates appears in Table 2 . $^{*}$ The rates are not inconsistent with the most recent CDF data, especially given that the latter show a somewhat larger number of events than expected for the favored mass, $m_{t} \simeq$ $170 \mathrm{GeV}^{[19]}$ However, the $t^{\prime}$ events do not necessarily provide a natural explanation for the excess; they contain significantly more jets and yield (following the CDF procedure) significantly lower reconstructed 'top' mass values. A thorough "second top" study by the CDF group would be most interesting.

The corner region of Fig. 1a, where $R_{b / \tau}$ and $R_{b^{\prime} / \tau^{\prime}}$ are nearest to 1 , contains the case $m_{t^{\prime}} \simeq m_{b^{\prime}} \simeq 110-120 \mathrm{GeV}$. Again, if $b^{\prime} \rightarrow c, u$ and $t^{\prime} \rightarrow b, s, d$ are adequately suppressed, then for $m_{t^{\prime}}>m_{b^{\prime}}$ the $t^{\prime}$ decays to $b^{\prime}$ along with a rather low mass system of $W$-decay products. The $b^{\prime}$ would then decay preferentially to $Z+$ light quark, giving a dilepton signal that would probably have been detected.

The above discussions would be altered should the light, neutral Higgs boson $(h)$ of the MSSM have mass less than $m_{b^{\prime}}$, since then $b^{\prime} \rightarrow h b$ decays would certainly (most probably) be dominant for $m_{b^{\prime}}$ smaller (greater) than $m_{Z}+m_{b}{ }^{[20]}$ The possibility of losing signals such as the above-noted dilepton channel cannot be discounted. Although at tree-level $m_{h}$ is small at the small $\tan \beta$ values allowed in the presence of a fourth generation, the latter can add substantially to the radiative corrections from the top/stop sector (depending upon superpartner masses).

From Figs. 1 and 2 we see that the RG equations also have solutions with $m_{t^{\prime}}<m_{b^{\prime}}<m_{t}$. Experimental constraints in this case depend on how the $t^{\prime}$ decays. We regard it as quite likely that $3 \mathrm{rd}-4$ th generation mixing will be large enough that the $t^{\prime} \rightarrow b W$ decays will be dominant. In this case, a $t^{\prime}$ with $m_{t^{\prime}}<130 \mathrm{GeV}$ (which limit applies for all $m_{t^{\prime}}<m_{b^{\prime}}$ regions allowed by the MSSM RGE's) has

** For comparison, at $m_{t}=170 \mathrm{GeV}$, we obtain an uncut $t \bar{t}$ cross section of $4 \mathrm{pb}$. Of the $\sim 80$ events predicted for $L=19.2 \mathrm{pb}^{-1}$, roughly 4 events pass our single-lepton plus $b$-tag cuts, and 0.8 events pass our dilepton cuts. 
been excluded by the normal $t$-quark searches. However, constraints could be much weaker if $3 \mathrm{rd}-4$ th generation mixing is extremely small and the $t^{\prime}$ decays were of the $\left(t^{\prime} \rightarrow c X\right)$ FCNC variety. For further details, see Ref. [24].

\section{Conclusions.}

In summary, our analysis has demonstrated that there is still room to add a fourth generation in the gauge-unified MSSM without violating perturbative or experimental constraints. However, the range of solutions is restricted even when $\lambda_{b} / \lambda_{\tau}$ and $\lambda_{b^{\prime}} / \lambda_{\tau^{\prime}}$ are unconstrained at $M_{U}$. In terms of running masses, if $m_{t}>150 \mathrm{GeV}$ the fourth generation $\tau^{\prime}$ and $\nu^{\prime}$ masses cannot lie above $86 \mathrm{GeV}$, and they can only be this large if $m_{t^{\prime}}, m_{b^{\prime}}$ are near the the LEP lower bound of $m_{Z} / 2$. For the quarks, $m_{t^{\prime}} \lesssim m_{t}$ if $m_{t} \gtrsim 170 \mathrm{GeV}$ - maximal $m_{t^{\prime}}$ is attained for minimal $m_{\nu^{\prime}}, m_{\tau^{\prime}}$, i.e. $\sim m_{Z} / 2$. Solutions with $m_{t^{\prime}} \sim m_{t} \sim 160-170 \mathrm{GeV}$ are possible for $\tan \beta \sim 2.2$; they require $m_{b^{\prime}}<m_{Z}$. Although our Monte Carlo study shows that these mass values could give rise to extra "top-like events", there is no immediate conflict with the current CDF top signal analysis. However, more data will greatly increase the constraints. For $m_{t} \sim 160-170 \mathrm{GeV}, \tan \beta \sim 1.5$ yields solutions with $m_{t^{\prime}} \gtrsim m_{b^{\prime}} \simeq 110-120 \mathrm{GeV}$. Such a case could be hidden in the data if $m_{t^{\prime}}-m_{b^{\prime}}$ is small enough that the $W^{*}$ decay products are very soft and if $m_{h}<m_{b^{\prime}}-m_{b}$ so that the $b^{\prime}$ decays mainly to Higgs boson plus $b$ quark. This latter case is also an example where $\lambda_{t, t^{\prime}, b^{\prime}}$ fixed-point behavior comes close to being achieved, and $\lambda_{b} / \lambda_{\tau}, \lambda_{b^{\prime}} / \lambda_{\tau^{\prime}}$ are large (choosing the relatively large value of $m_{b}\left(m_{b}\right)=4.6 \mathrm{GeV}$ ). For still smaller $\tan \beta(\sim 1.2)$, one can approach even nearer a $\lambda_{t, t^{\prime}, b^{\prime}}$ fixed point and obtain $\lambda_{b} / \lambda_{\tau} \gtrsim 0.65$ and $\lambda_{b^{\prime}} / \lambda_{\tau^{\prime}} \sim 1$. However, the corresponding $m_{b^{\prime}}, m_{t^{\prime}}$ values are typically such that $m_{b^{\prime}}>m_{t^{\prime}}$ and $m_{t^{\prime}}<m_{Z}$, a scenario that would have escaped detection only if the $t^{\prime} \rightarrow b W^{(*)}$ decays are unexpectedly suppressed.

To "tighten the noose" on the fourth generation, a variety of theoretical and experimental questions should be pursued. For example, how is electroweak symmetry breaking affected, and how do detailed fits of precision electroweak data change when $N_{g}=4$ ? We believe that our study has shown that these questions are of considerable theoretical and experimental interest, and should be explored further.

\section{Acknowledgments}

We wish to thank R. Blair and J. Huston for communicating the status of their $b^{\prime}$ analysis to us. DWM thanks the U.C. Davis Department of Physics for its hospitality during his '93-'94 sabbatical. This work has been supported in part by Department of Energy grants \#DE-FG03-91ER40674, \#DE-FG02-85ER40214, and by Texas National Research Laboratory grant \#RGFY93-330. 


\section{References}

1. P. Langacker, in Proceedings of the PASCOS-90 Symposium, eds. P. Nath and S. Reucroft (World Scientific, 1990); P. Langacker and M. -X. Luo, Phys. Rev. D44, 817 (1991); U. Amaldi, W. de Boer, and H. Furstenau, Phys. Lett. B260, 447 (1991); J. Ellis, S. Kelley and D. V. Nanopoulos, ibid. 260, 131 (1991).

2. S. Dimopoulos, L. Hall and S. Raby, Phys. Rev. Lett. 68, 1984 (1992); Phys. Rev. D45, 4192 (1992); P. Ramond, R.G. Roberts and G. Ross, Nucl. Phys. B406, 19 (1993).

3. V. Barger, M. S. Berger, T. Han and M. Zralek, Phys. Rev. Lett. 68, 3394 (1992); H. Arason, D. J. Castano, E. J. Piard and P. Ramond, Phys. Rev. D47, 232 (1993); V. Barger, M. S. Berger and P. Ohmann, Phys. Rev. D47, 1093 (1993); C. Albright and S. Nandi, Fermilab-PUB-93/316-T, OSU-Preprint 282; G. L. Kane, C. Kolda, L. Roszkowski and J. Wells, UM-TH-93-24, Oct. 1993.

4. P. Agrawal and W. -S. Hou, Phys. Rev. D46, 1022 (1992); P. Agrawal, S. Ellis and W. -S. Hou, Phys. Lett. B256, 289 (1991); S. King, ibid,281, 295 (1992); C. Hill and E. Paschos, ibid., 241, 96 (1990); W.-S. Hou and R. G. Stuart, Nucl. Phys. B349, 91 (1991); M .Sher and Y. Yuan, Phys. Lett. B285, 336 (1992); H. Fritzsch, Phys. Lett. B289, 92 (1992); B. Mukhopadhyaya and D.P. Roy, Phys. Rev. D48, 2105 (1993).

5. Mark II Collaboration, G. S. Abrams et al., Phys. Rev. Lett. 63, 2173 (1989); L3 Collaboration, B. Advera et al., Phys. Lett. B231, 509 (1989); OPAL Collaboration, I. Decamp et al, ibid., 231, 519 (1989); DELPHI Collaboration, M. Z. Akrawy et al. ibid., 231, 539 (1989).

6. S. King, Ref. [4].

7. M. Chanowitz, J. Ellis and M. Gaillard, Nucl. Phys. B128, 506 (1977); A. Buras, J. Ellis, M. Gaillard and D. V. Nanopoulos, ibid., 135, 66 (1978); M.B. Einhorn and D.R.T. Jones, ibid., 196, 475 (1982); J. Ellis, D. V. Nanopoulos and S. Rudaz, ibid., 202, 43 (1992); a recent analysis of both SM and the MSSM is given in H. Arason, D. J. Castano, E.J. Piard and P. Ramond, Phys. Rev. D47, 232 (1990).

8. D. V. Nanopoulos and D. Ross, Phys. Lett. B118, 99 (1982); J. Bjorkman and D. Jones, Nucl. Phys. B259, 533 (1985).

9. P. Langacker and N. Polonsky, Phys. Rev. D47, 4028 (1993); ibid., 49, 1454 (1994); V. Barger, M. Berger, P. Ohmann, and R. J. N. Phillips, Phys. Lett. B314, 351 (1993); G. Kane et al. Ref. [3]; G. Ross and R.G. Roberts, Nucl. Phys. B377, 571 (1992); B.D. Wright, MAD/PH/812. For string Yukawa threshold effects, see I. Antoniadis, E. Gava, K.S. Narain and T.R. Taylor, Nucl. Phys. B407, 706 (1993). 
10. In J. L. Lopez, D. V. Nanopoulos and A. Zichichi, CERN-TH-713 8/94, $0.7<\lambda_{b}\left(M_{U}\right) / \lambda_{\tau}\left(M_{U}\right)<1$ is studied at the string scale.

11. P.H. Frampton, S. Nandi and J.G. Scanio, Phys. Lett. B85, 225 (1979).

12. B. Pendelton and G. Ross, Phys. Lett. B98, 291 (1981); C. Hill, Phys. Rev. D24, 691 (1981); W. Bardeen, M. Carena, S. Pokorski and C. Wagner, Phys. Lett. B320, 110 (1994); J. Bagger, S. Dimopoulos and E. Masso, Phys. Rev. Lett. 55, 920 (1985).

13. K. Inoue, A. Kabuto, H. Komatsu, and S. Takeshita, Prog. Theor. Phys. 67, 1889 (1982); Bjorkman and Jones, Ref. [9].

14. See P. Langacker and N. Polonsky, Ref. [9].

15. V. Barger, M. S. Berger and P. Ohmann, Ref. [5].

16. OPAL Collaboration, M. Z. Akrawy et al. Phys. Lett. B236, 364 (1990); ALEPH Collaboration, D. Decamp et al., ibid., 236, 511 (1990).

17. Particle Data Group, K. Hikasa et al., Phys. Rev. D45, 51 (1992), vol. 11, pp. 159-163.

18. F. Abe et al., Phys. Rev. Lett. 64, 147 (1990), Phys. Rev. Lett. 68, 447 (1992). T. Trippe, Particle Data Group, private communication.

19. F. Abe et al., Fermilab-PUB-94/097-E.

20. W. -S. Hou and R. Stuart, Phys. Rev. D43, 3669 (1991).

21. See Agrawal and Hou, and Agrawal, Ellis and Hou, and references therein, Ref. [4].

22. J. Houston and R. Blair, private communication.

23. D0 Collaboration, S. Abachi et al., Phys. Rev. Lett. 72, 2139 (1994).

24. Work in progress. 